\title{
Tactile Texture Recognition with a 3-Axial Force MEMS integrated Artificial Finger
}

\author{
Florian de Boissieu, Christelle Godin, \\ Bernard Guilhamat and Dominique David \\ CEA, LETI, MINATEC \\ 17 rue des Martyrs \\ 38054 Grenoble Cedex 9 \\ France
}

\begin{abstract}
Recently, several three-axial MEMS-based force sensors have been developed. This kind of force micro sensor is also called tactile sensor in literature for its similarities in size and sensitivity with human mechanoreceptors. Therefore, we believe these three-axial force sensors being able to analyse textures properties while sliding on a surface, as would do a person with his finger. In this paper, we present one of these sensors packaged as an artificial finger, with a hard structure for the bone and a soft rubber for the skin. Preliminary experiments show a good sensitivity of the finger, as its ability to sense the periodic structure of fabrics or to differentiate papers from fabrics calculating a friction coefficient. Its performance for discrimination of different surfaces is then estimated on fine textures of 10 kinds of paper. Supervised classification methods are tested on the data. They lead to an automatic classifier of the 10 papers showing good performances.
\end{abstract}

\section{INTRODUCTION}

Research on tactile sense has stirred up a growing interest in the past few years. Giving a robot the perception of forms and textures would open to lots of applications, as object manipulation or objective texture recognition, in fields as different as paper and fabric manufactures, surgery [1] or cosmetics [2].

During the ten last years, several artificial finger prototypes were developed for texture recognition. To reproduce texture sensing, an approach is to develop sensors by mimicking the structural features of a human finger. Howe [3] and Tanaka [4] developed sensors composed of several layers of different elasticity to imitate the bone and the layers of skin (dermis, epidermis...). Mechanoreceptors were represented by strain gauges and piezoelectric elements as PVDF (polyvinylidene fluoride) integrated in the artificial skin. Howe manages to detect a $6.5 \mu \mathrm{m}$ high knife-edge probe. Tanaka discriminates rubbers of different hardness and roughness (450-100 $\mu \mathrm{m}$ grain size). Mukaibo [5] went a little further adding skin ridges to its artificial finger, as well as a cantilever system measuring the overall normal and shear forces. Hosoda [6] integrated two kinds of receptor, strain gauges and PVDF films, in the two layers of an anthropomorphic fingertip, reproducing by this way the four kinds of mechanoreceptors of human fingertips. These two last prototypes integrate mono-axial strain sensors and the studies were limited to differentiate textures of quite

\author{
Christine Serviere and Daniel Baudois \\ GIPSA-Lab \\ 961 rue de la Houille Blanche \\ Domaine universitaire - BP 46 \\ F - 38402 Saint Martin d'Heres cedex \\ France
}

different materials (cork, aluminium, wood...). Moreover, these prototypes size is quite large compared to a human finger. This can be a problem for finer texture studies.

Recently, several three-axial MEMS (Micro Electro Mechanical Systems) measuring forces in the three dimensions of space have been developed [7], [8], [9], [10]. Except Beccai et al. [11], who report some slip detection results with a tactile device for an artificial hand, very little results have been published on the use of three-axial MEMS force sensor in artificial tactile sensing experiments. On the basis of Yao's model [12], CEA-LETI is producing one of these innovative sensors as individual elements and as arrays of $10 \times 1$ and $10 \times 3$ elements.

Among the four kinds of mechanoreceptors we own in the fingertip, Pacinian Corpuscules (PC) are responsible for the detection of vibrations occuring while rubbing a surface. The size of a PC is about $1 \mathrm{~mm}$ large and its sensitivity about 1 $\mathrm{mN}$. As it is implanted quite deeply in the skin, its receptive field is large. But what makes the PC interesting is its high frequency response $(15 \mathrm{~Hz}-400 \mathrm{~Hz})$ and therefore its capability to detect small vibrations coming from fine texture exploration [13].

The sensor produced by CEA-LETI is similar in size and sensitivity to those of a PC. As it is a silicon MEMS it has a high frequency response. Our approach is to use such a sensor to study fine textures discrimination. We integrated this silicon MEMS to a finger shaped structure and covered it with a soft rubber skin to protect it. We expect this artificial finger to be able to discriminate fine textures exploring surfaces as would do a human person.

The first part of this paper describes the sensor and the tactile exploration system. The second part presents two preliminary experiments to state on the sensitivity of the sensor: the discrimination of coarse textures such as paper and fabric, and the reconstruction of forces and friction coefficient images exploring a printed paper. The last part describes an experiment of fine texture classification for 10 different kinds of paper. This document presents the results of two classification algorithm among several tested. First, a supervised classification method, using spectral features, gave us a classification rate of $60 \%$, which is good compared 

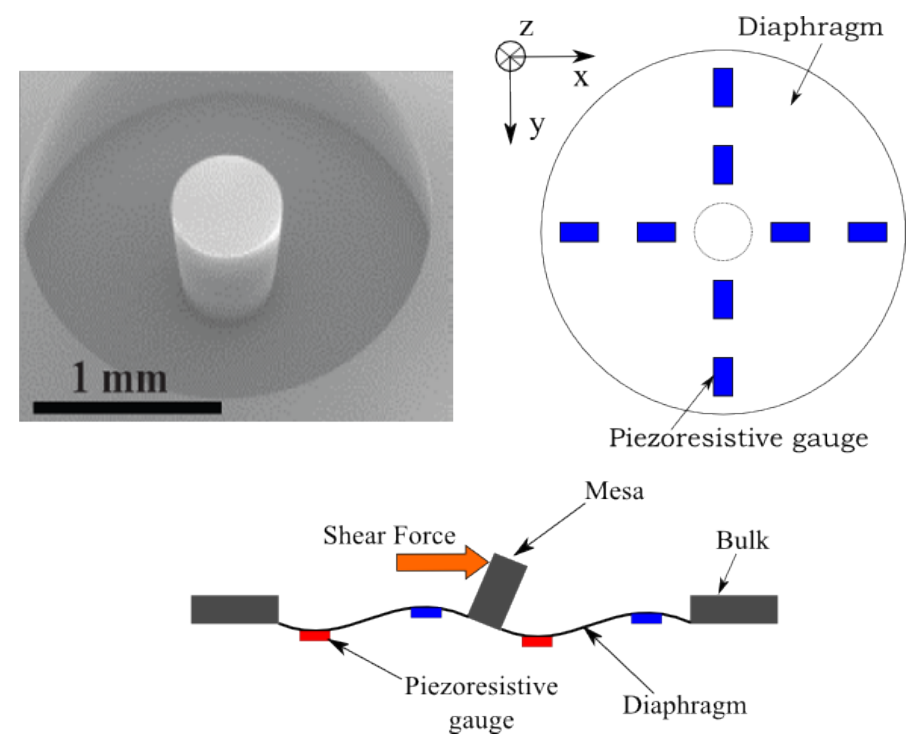

Fig. 1. Three-axial sensor made in a silicon mono-crystal. Top,Left: Scanning Electrnical Microscopy (SEM) photo of the three-axial sensor. Top, Right: bottom view of the diaphragm. Bottom: Diaphragm deflection when a force is applied. Here, red gauges are stretched by the deflection, blue gauges are compressed.

to the $10 \%$ random classification rate. The second applying neural networks shows similar results but using only 5 selected features.

\section{ARTIFICIAL FINGER AND EXPLORATION SYSTEM}

\section{A. Artificial finger}

The artificial finger has three components: a hard structure for the bone, a tactile element for the mechanoreceptor and a rubber for the artificial skin.

The silicon force sensor (see figure 1) is a sensor measuring stress in three dimensions of space. It consists of a monocristal silicon element, composed of a small mesa surrounded by a thin diaphragm under which 8 piezoresistors are diffused. The piezoresistors are implanted on orthogonal axis $\mathrm{x}$ and $\mathrm{y}$. As a force is applied on the mesa, the diaphragm will deflect. The resulting strain of the diaphragm induces a compression or a stretch of the piezoresistors, changing their resistor values. Connecting these resistors to Wheatstone bridges, we can measure three voltages $U_{x}, U_{y}, U_{z}$ respectively proportional to the three components $F_{x}, F_{y}, F_{z}$ of an equivalent force on the mesa as demonstrated in [12]. Figure 1 (bottom) illustrates the strain of the diaphragm when a force is applied on the mesa. This silicon sensor is able to support a $1 M P a$ pressure and a $2 N$ tangential force on top of the mesa.

The silicon tactile sensor is flush-mounted on a cylindrical piece of hard epoxy and wire-bonded to its electronic. The electronic is composed of three amplifiers, one for each output voltage.

To protect the silicon sensor and also to transmit friction forces when exploring a texture, the sensor is covered by a soft rubber skin made of polyurethane as shown in figure 2. The rubber shape is composed of two parts: a cylindrical

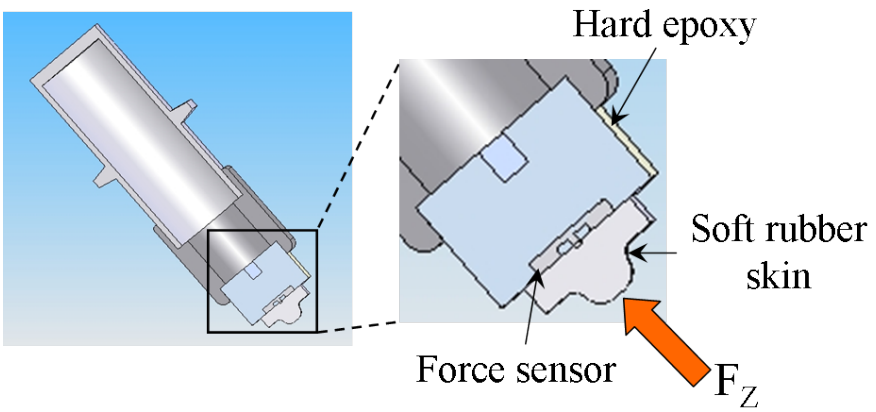

Fig. 2. Artificial finger composed of the 3-axial force sensor flush-mounted on a hard epoxy layer and covered by a soft rubber skin

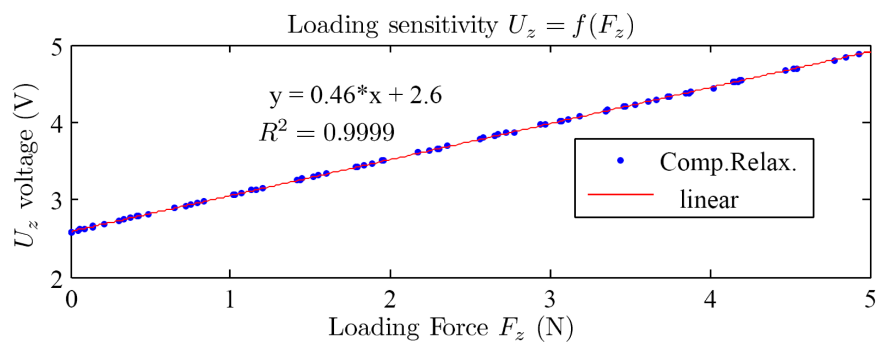

Fig. 3. Loading response of the artificial finger covered with 80 shore A rubber, for three loading-unloading cycles.

basis over the silicon sensor and a semi-spherical top part that will be in contact with the sample to be explored. The semi-spherical shape of the top part was designed to keep the contact surface quite constant even with the wear. The size of this top part was chosen to keep a contact surface small enough $(\phi \simeq 3 \mathrm{~mm})$ to detect texture details such as fine roughness and small friction events of textures. The basis of the rubber, which is in contact with the silicon sensor, is chosen just a little larger than the size of the silicon diaphragm, so that it optimizes the measurement of the global force applied at on the top when exploring a texture. At first the rubber was made of polyurethane AXSON UR5801/UR5850 of hardness 50 shore A. This rubber was used for preleminary experiments presented in section III. But as it weared out fast, we decided to change it for a polyurethane LOCTITE 3957 of hardness 80 shore A. This rubber is hard enough to limit considerably the wear, but it is still soft enough to keep a contact surface sufficient to be sensitive to friction without deteriorating paper or fabrics samples while rubbing them. The 80 shore A rubber was used for the fine texture classification experiment presented in section IV. The artificial finger covered with poluyrethane was characterized in loading, showing a very good linearity with both kind of rubber. Figure 3 presents the loading characteristic of the sensor covered with the 80 shore A rubber.

\section{B. Exploration and acquisition systems}

The artificial finger is fixed on the dart of an HP plotter machine (sort of printer) used as the sample exploration system (see figure 4). The plotter has two step motors allowing to 


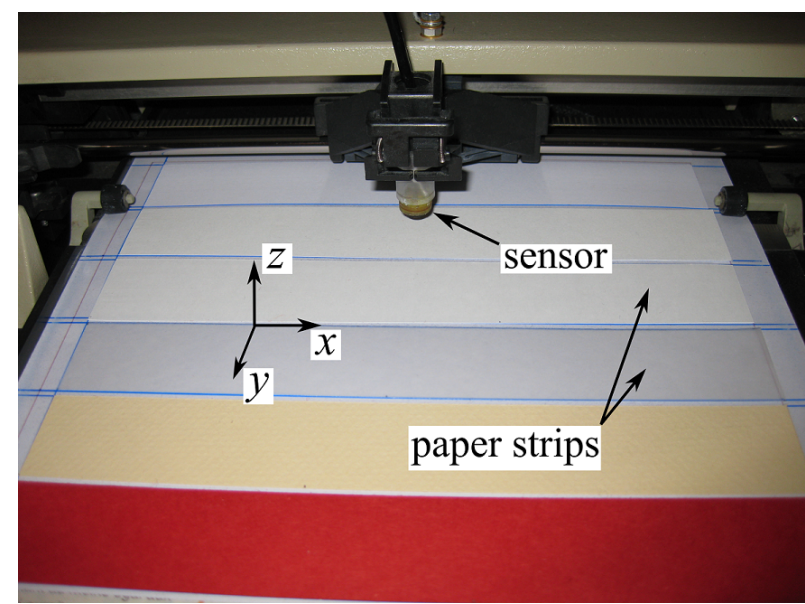

Fig. 4. Plotter machine used as the exploration system. The artificial finger is fixed on the dart usually dedicated to take a pencil. On this picture, the last experiment paper samples presented in section IV is installed on the plotter.

explore surfaces in both $\mathrm{x}$ and $\mathrm{y}$ directions. One is dedicated to move the sensor along a bar representing the $\mathrm{x}$-axis. The other one is used to move the samples along the y-axis thanks to rollers. An electromechanical system lifts up the sensor when not exploring. A $0.4 \mathrm{~N}$ normal force is applied thanks to a string on the electromechanical system. The plotter is connected to a PC workstation by a National Instrument GPIB-USB cable in order to control the moving speed and the position of the artificial finger. The acquisition system is composed of an electronic circuit of amplification, an analogue Nyquist filter (SCXI 1000 Nat. Inst.) and a data acquisition card (DAQCard 6036E Nat. Inst.). The exploration and acquisition systems are synchronously controlled by a dedicated Labview software.

\section{PRELIMINARY EXPERIMENTS}

To state on the sensitivity of the artificial finger two experiments were built up as a preliminary to the classification experiment. The first experiment aims at testing the ability of the sensor to discriminate coarse textures easily discriminable by touch. The purpose of the second experiment is to reconstruct an image of the forces felt by the artificial finger when exploring a printed paper.

\section{A. Coarse texture discrimination}

In this experiment four samples were explored: two papers and two fabrics. The papers (P1 and 2) have a special texture called Soft Touch (ArjoWiggins) which is quite soft and grips a little. The samples of fabric are both made of cotton, but each one has its own periodic weave form: one with a jean weave (T1), the other with a plain weave (T2). The experiment consisted in exploring the four samples in $x$ and $y$ directions with two different speeds $(v=3.8 \mathrm{~cm} / \mathrm{s}, 7.6 \mathrm{~cm} / \mathrm{s})$.

Firstly, observing the spectrum of $U_{x}$ voltage for fabrics, we detect easily the lines corresponding to the periodic weave forms. Figure 5 presents a photo of the jean texture and the corresponding spectrum of an $x$-direction exploration. We

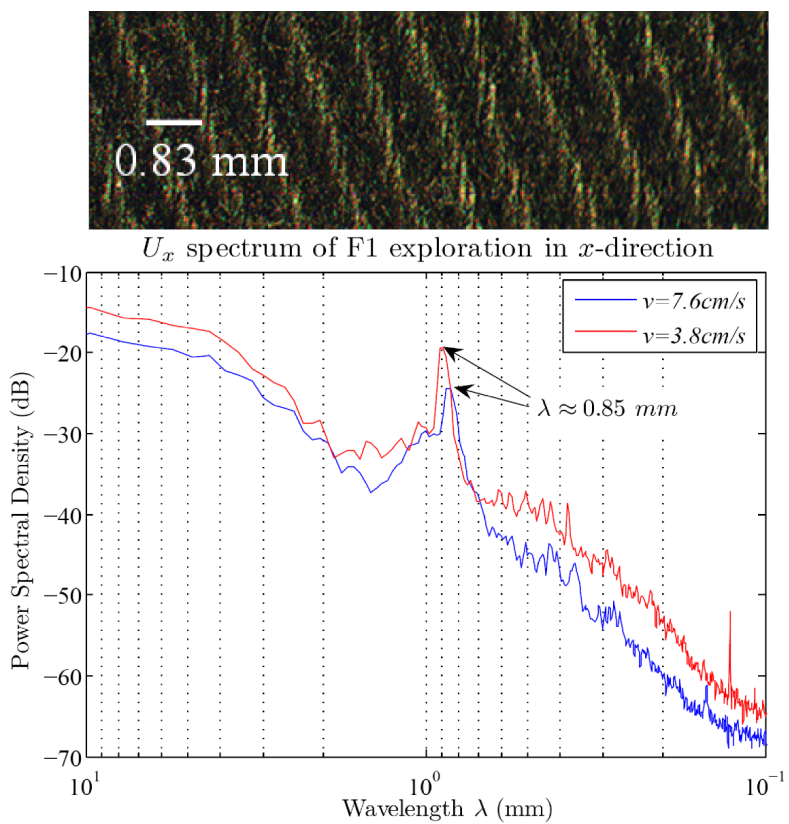

Fig. 5. Top: Jean texture (F1) photo. In $x$-direction the Jean weave is about $0.83 \mathrm{~mm}$ periodic. Bottom: Spectrum of $U_{x}$ voltage for $v=3.8 \mathrm{~cm} / \mathrm{s}$ in red and $v=7.6 \mathrm{~cm} / \mathrm{s}$ in blue. The common line $(\lambda \approx 0.85 \mathrm{~mm})$ for both speed exploration spectra corresponds to the weave periodicity. The extensibility and the deformability of the material explain the difference between theoretical and measured wavelength.

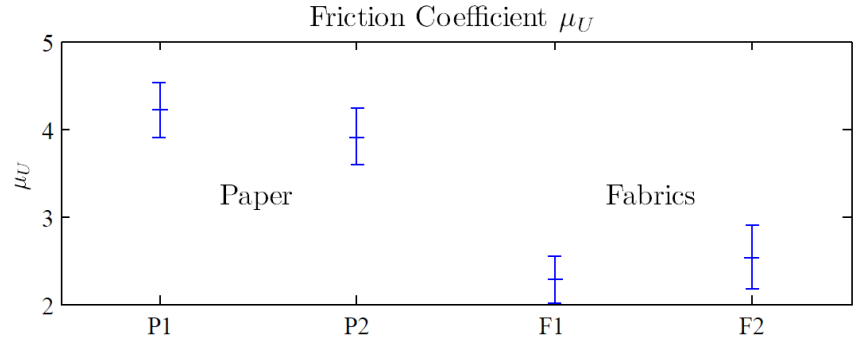

Fig. 6. Friction coefficient $\left(\mu_{U}\right)$ of paper samples (P1, P2) and fabrics samples (F1, F2).

can note the common line for both speed of exploration corresponding to the weave wavelength $(\lambda \approx 0.85 \mathrm{~mm})$.

Secondly, we calculated, for each sample, a friction coefficient defined by $\mu_{U}=\frac{\sqrt{U_{x}^{2}+U_{y}^{2}}}{U_{z}}$, that we consider to be proportional to the usual friction coefficient defined by $\mu_{F}=$ $\frac{F_{T}}{F_{N}}$, where $F_{T}$ and $F_{N}$ are the tangential and normal forces at the contact surface. We find a higher friction coefficient for the Soft Touch papers than for the fabrics. The difference in the friction coefficients seems quite representative of the difference in stickiness we feel rubbing Soft Touch paper and fabric samples. Figure 6 shows the mean friction coefficient with bars representing standard deviation.

\section{B. Image of forces}

The second preliminary experiment consists in reconstructing the images of forces during the exploration of a texture. With images of forces we could expect a better visualization of 

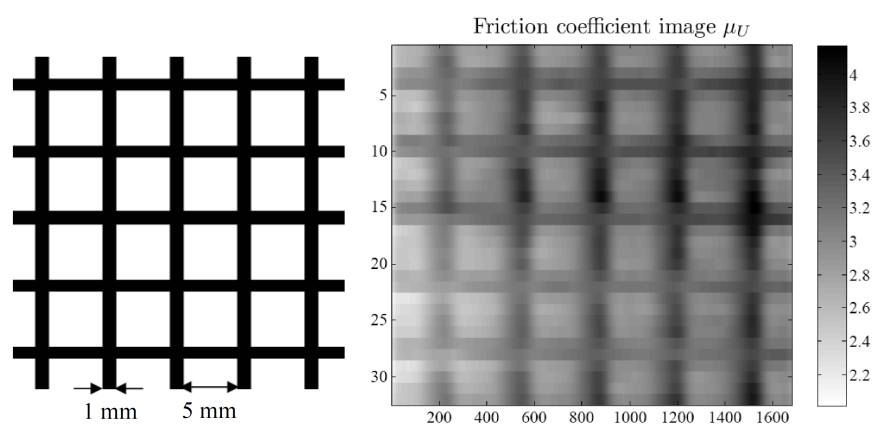

Fig. 7. Left: Original printed grid explored horizontally ( $x$-direction) at $v=38 \mathrm{~mm} / \mathrm{s}$. Right: $1680 \times 32$ pixels image of the friction coefficient $\mu_{U}$. The friction coefficient is greater for inked zones than for clean zones.

what is occurring to the sensor during exploration. The sample explored was a grid printed on white paper with a classical laser printer. The thickness of the ink layer was estimated, with an optical profilometer, to be of the order of $10 \mu \mathrm{m}$. Figure 7 (left) shows the dimensions of the printed grid.

The grid was scanned by the artificial finger on 32 lines in $x$-direction finger with a speed $v=38 \mathrm{~mm} / \mathrm{s}$ and a sampling frequency of $2 \mathrm{kHz}$. Scanned lines are spaced of $1 \mathrm{~mm}$. After slightly low filtering signal to avoid artefacts, we reconstructed a $1680 \times 32$ pixels image of friction coefficient $\mu_{U}$. Figure 7 (right) presents the reconstructed image of the friction coefficient. The image shows the great sensitivity of the sensors and the good repeatability of measures. We can note a greater friction coefficient for inked zones than for clean zones.

\section{Fine TEXTURE CLASSIFICATION}

Both preliminary experiments show a good sensitivity of the sensor and an easy discrimination of coarse and periodic textures. The first preliminary experiment shows it is easy to discriminate papers from fabrics with the friction coefficient. But it seems quite difficult on figure 6 to discriminate one paper from the other. The next step of this study was then to evaluate the ability of the artificial finger to discriminate quite similar, fine and random textures, like paper textures.

This new experiment consists in classifying 10 textures of paper, each texture of paper representing a class. We constructed an important database to evaluate the efficiency of several classification algorithms. One simple and fast algorithm using spectrum of $U_{x}$ gives rise to good results. The second presented below reduces signal to 5 features and uses a neural network as classification algorithm.

\section{A. Samples and database}

The study of classification was based on paper samples because it is particularly adapted to our exploration system and it offers lots of possibilities in terms of texture. Paper is also a material we are used to manipulate. For this classification experiment we selected 10 samples that we could identify relatively easily blind-rubbing them (easy for experts but much more difficult for a common person). Among the 10 paper
TABLE I

10 PAPER SAMPLES TO BE CLASSIFIED. EACH SAMPLE IS ASSOCIATED WITH A CLASS NUMBER ARBITRARLY CHOSEN

\begin{tabular}{|c|c|}
\hline Class n & Paper sample \\
\hline 1 & Printer paper \\
\hline 2 & Soft Touch \\
\hline 3 & Skin Touch \\
\hline 4 & Tracing paper \\
\hline 5 & Large grain size drawing paper \\
\hline 6 & Blotting paper \\
\hline 7 & Newspaper \\
\hline 8 & Coated paper \\
\hline 9 & Small grain size drawing paper \\
\hline 10 & Photo paper \\
\hline
\end{tabular}

samples, 8 are found in everyday life: newspaper, magazines (coated paper), printer paper, tracing paper, drawing paper with two grain size, photo paper and blotting paper. The last two papers are special Soft Touch and Skin Touch textures (ArjoWiggins), which are soft and grip a little. An arbitrarly chosen class number is associated to each paper sample, as shown in Table I.

The experiment consists in scanning the samples in different zones along one direction, here $\mathrm{x}$-axis of the plotter. The wear of the artificial finger can be considered as insignificant when sliding on a few centimetres. But for the experiment, the artificial finger is sliding on a total distance of $20 \mathrm{~m}$ (2 $\mathrm{m}$ on each sample) making the wear more important. To make following classification independent of the wear of the artificial finger rubber, the paper samples must be explored randomly. The trick we found to achieve this goal easily and to avoid any manipulation of samples during the experiment consisted in placing 10 paper strips of $25 \times 185 \mathrm{~mm}$, one of each sample, on the same support (see figure 4). For the support, an A4 sheet of transparency is chosen for its non-compressibility and smoothness, so that it doesn't disturb the sample perception. The strips are fixed along the $\mathrm{x}$-axis with a $3 \mathrm{M}$ spray adhesive repositionable that won't penetrate the samples. Thanks to its rollers, the plotter can move the transparency support along the y-axis and choose randomly the sample to be explored without any manipulation.

The sample exploration method consists in scanning $4 \mathrm{~cm}$ segments on the sample, along the $x$-direction, with the artificial finger. The samples were explored at a speed of $3.8 \mathrm{~mm} / \mathrm{s}$ with a sampling frequency of $1 \mathrm{kHz}$. Compared to previous experiments, the speed of exploration was reduced in order to reduce rubber wear. The samples are randomly scanned 50 times each resulting in a database of 50 acquisitions of $\left(U_{x}, U_{y}, U_{z}\right)$ per class.

\section{B. Feature selection}

Among the three voltage components, $U_{x}$ (measuring the force $F_{x}$ parallel to the movement) seemed to be the more relevant since $U_{y}$, measuring the force $F_{y}$ perpendicular to the movement, is quite reduced, and $U_{z}$, measuring the loading force $F_{z}$ controlled by the string, is almost constant. Hence, 


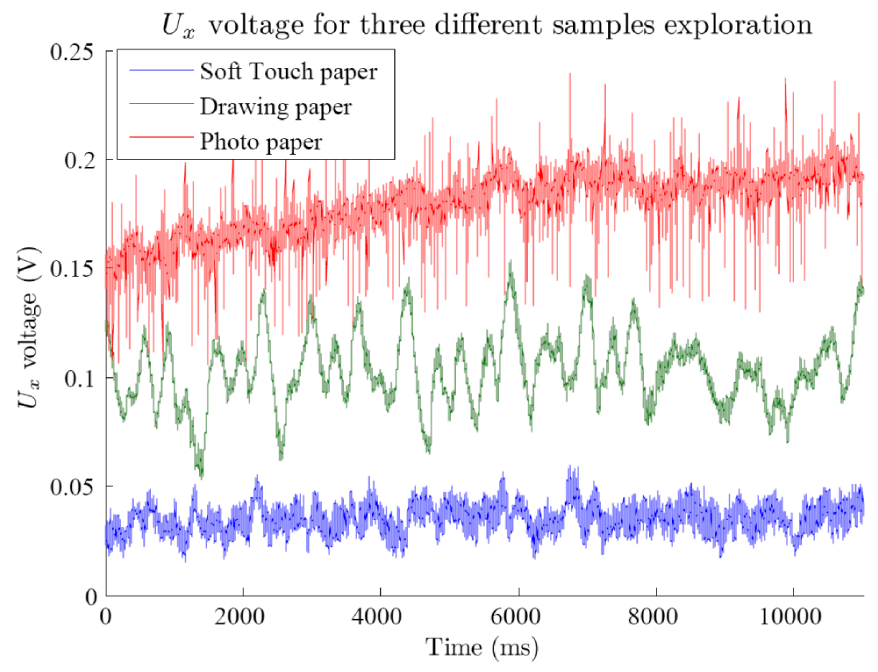

Fig. 8. $U_{x}$ voltages for the exploration of Soft Touch paper, grained drawing paper and photo paper. These signals illustrate the differences found in friction coefficient, variance and kurtosis features which can be identified respectively as easiness in sliding, texture roughness and stick-slip.

to simplify the analysis, we restricted ourselves to the use of $U_{x}$ for this classification study.

To identify characteristic features of the sample explored, signals are analysed in the complementary temporal and spectral domains. Three features were found to be characteristic of the samples in the temporal domain:

- Friction coefficient $\mu_{U}$ : for a constant normal force $\mu_{U}$ is identified as the mean tangential force necessary to slide.

- $U_{x}$ variance $\sigma_{x}^{2}$ representing the variations of forces around its mean. It can be identified as a representation of texture roughness.

- $U_{x}$ kurtosis (4th statistical order) measuring the peackedness of a distribution. A gaussian distribution has a kurtosis value of 3 . A flat distribution is giving a kurtosis value below 3 and a peack distribution a kurtosis value over 3. The kurtosis can be interpreted as a measurement of the amount of infrequent extreme deviations of the signal, as opposed to frequent modestly-sized deviations measured by variance. We identify the kurtosis as a measure for the stick-slip effect occurring when exploring a flat adherent surface, as photo paper.

Figure 8 presents $U_{x}$ signals of Soft Touch paper (blue), grained drawing paper (green) and photo paper (red). This figure illustrates well the three features presented above. Figure 9 presents the mean temporal features of the 10 classes sorted in ascending order. Soft touch paper (class 2) is soft but grips a little. Its corresponding signal, blue signal on figure 8 , has a low $U_{x}$ mean and a quite important density of small peaks. This is characterized by a low friction coefficient but a kurtosis value slightly over 3, as shown on figure 9 . Grained drawing paper (class 5) has an important roughness which is characterized by an important variance (important $U_{x}$ variations on green signal). Photo paper (class 10) is very adherent which can be seen on red signal as a high
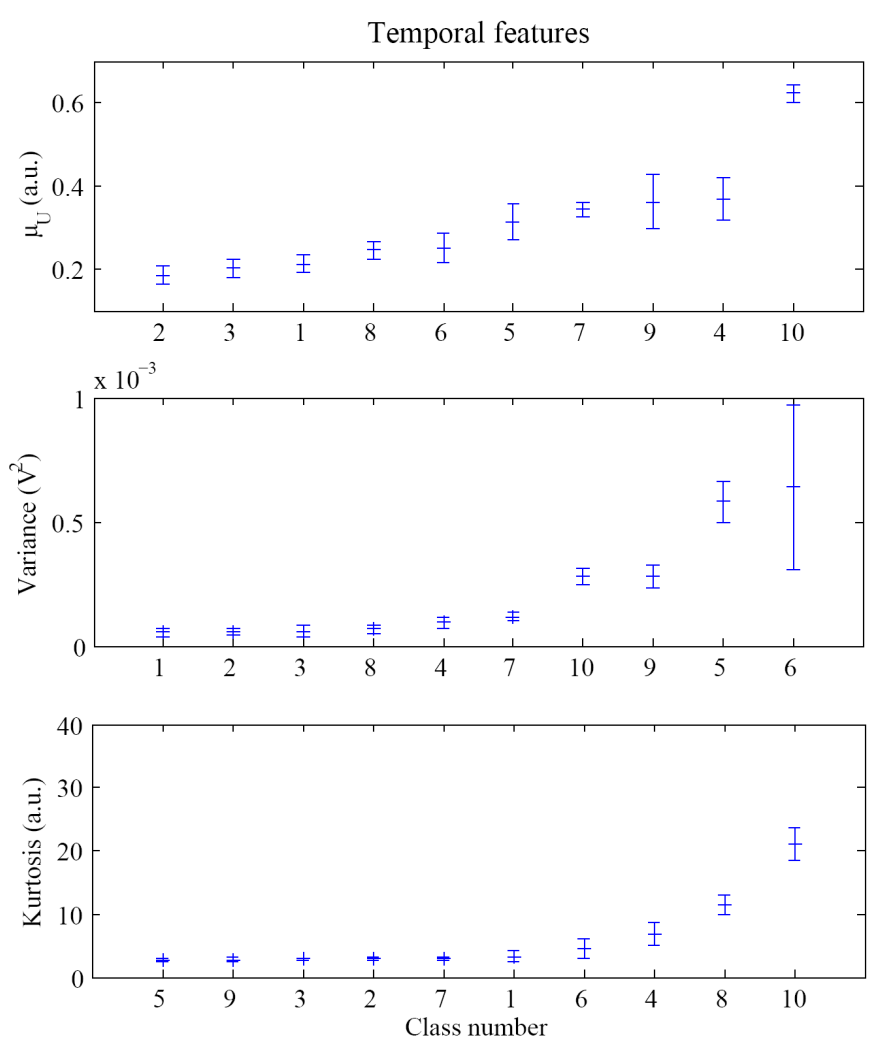

Fig. 9. Mean values of the three temporal features, friction coefficient, variance and kurtosis, versus class number. The mean values are calculated on the training data. Bars represent the intra-class standard deviation of the feature. Classes are sorted in ascending order. One can note that the friction coefficient is almost 10 times smaller than the one presented in figure 6 . It could be attributed to the 80 shore A rubber that is about 10 times harder than the 50 shore A rubber used in previous experiments. The 80 shore A rubber slips much easier on the paper surfaces giving a lower tangential force for the same normal force applied.

density of big peaks. It shows the stick-slip characteristic of the movement. Therefore, it has an important kurtosis value. On figure 8 the discrimination between the three samples seems easy. But as one can see on figure 9, some classes are overlapping in feature space making the classification much more difficult.

The spectrum of each acquisition was extracted from the $U_{x}$ component. As the plotter exploration system was quite noisy, due to the step motors, we extracted the band $20-250 \mathrm{~Hz}$ from the spectrum and filtered out motors vibrations. Figure 10 presents the preprocessed spectra of Soft Touch, drawing and photo paper samples converted in decibel. On this figure we can observe three features that seem to differ from a sample to another:

- the decibel spectrum mean

- the decibel spectrum slope

- the decibel spectrum form, that is to say the resonances

Figure 11 illustrates the mean spectral features of the 10 classes sorted in ascending order. Paper samples submited to a stick-slip movement are showing a higher spectrum mean and a lower spectrum slope than the other classes of samples. 


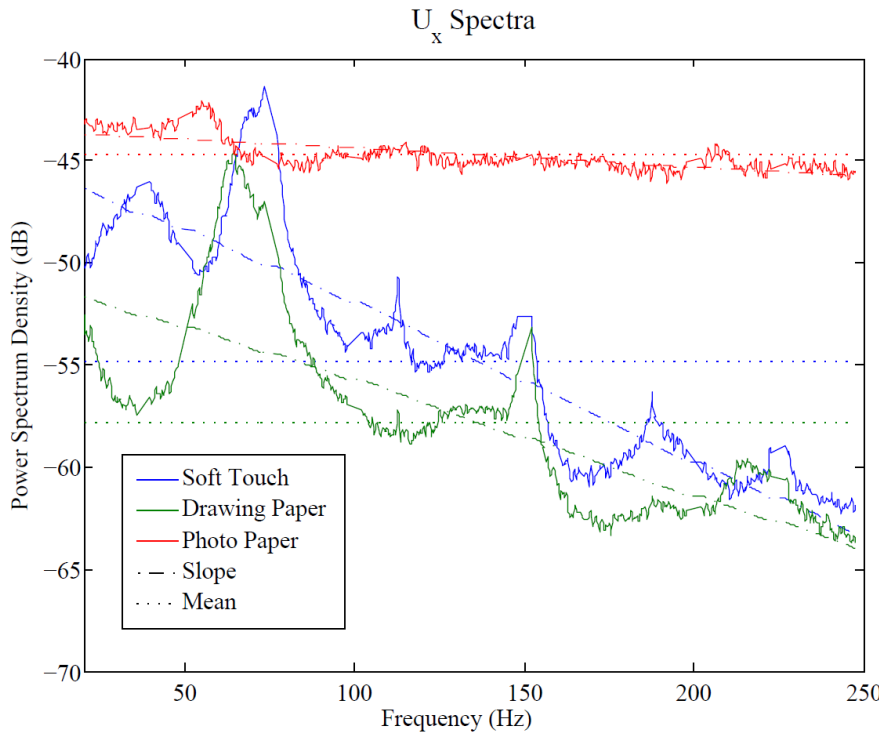

Fig. 10. Spectrum, spectrum slope and spectrum mean of Soft Touch, drawing and photo paper samples.
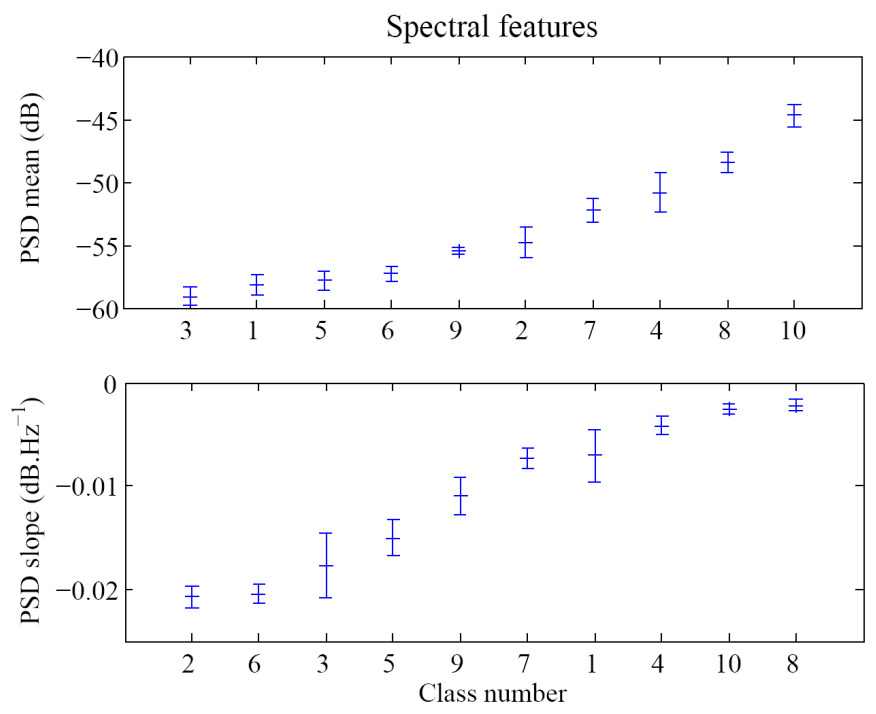

Fig. 11. Mean values of the two spectral features, spectrum mean and slope, versus class number. The mean values are calculated on the training data. Bars represent the intra-class standard deviation of the feature. Classes are sorted in ascending order.

\section{Classification algorithms}

The data features are of two types. On one side the 5 features that are the friction coefficient, variance, kurtosis, spectrum slope and mean. On the other side the spectrum form that is composed of 822 frequency coefficient. Therefore, two classification algorithms were tested to classify the ten paper samples. The first consists in classifying the spectrum forms with a minimum euclidean distance to the mean spectrum. The second uses the 5 features left as an input to an artificial neural network. This last method permits to reduce the space of classification inputs and to take into account non-linearities. The following is presenting these two methods.
1) Minimum euclidean distance to mean spectrum: The generic classification method usually consists in training a model for each class, comparing a test sample to the models via a criterion and, according to the result, deciding if it belongs to a class or another. Here, the training method is doing the mean characteristic of each class, which is expressed as follows:

$$
P^{j}(f)_{d B}=10 \cdot \log _{10}\left(\sum_{i=1}^{N} S_{i}^{j}(f)\right)
$$

where $P^{j}(f)_{d B}$ is the mean of $N$ spectra of class $j$ converted in decibel and $S_{i}^{j}(f)$ is the spectrum of the $i^{t h}$ acquisition belonging to class $j$. As the form of the spectrum seemed to contain most of the discriminative information between samples, the criterion chosen was the minimum euclidean distance to the mean spectrum, where the euclidean distance between two spectra $S_{i}$ and $S_{j}$ is defined as:

$$
d\left(S_{i}, S_{j}\right)=\sqrt{\sum_{f}\left(S_{i}(f)_{d B}-S_{j}(f)_{d B}\right)^{2}}
$$

Hence the decision is given by:

$$
C=\min _{j}\left[d\left(S, P^{j}\right)\right]
$$

where $S$ is a spectrum to be classified and $C$ the attributed class.

2) Neural Network algorithm: Multi-layer perceptron (MLP) neural networks are known to be well suited for non-linear classification problems. But its structure must be chosen carefully. For multi-class classification tasks several configuration can be tested. The configuration that gave us the best results was a one-against-rest classification method. That is to say 10 MLPs, one for each class, each MLP having a unique output neuron with target 1 for its associated class examples and 0 for others. Each MLP is composed of a 5 inputs layer, one input for each of the 5 features, which distributes the data to a hidden layer itself connected to an output neuron. A log sigmoid transfer function is chosen for both hidden layer and ouptut neurons, giving then an output in the interval $[0 ; 1]$. Between the 10 outputs of the 10 MLPs, the class attributed is the class associated with the MLP having the greatest output. To avoid overfitting, we decided to restrict to a hidden layer with a maximum of 5 neurons.

The weights of the networks are trained using a Bayesian regularization backpropagation algorithm. This algorithm trains fast and produces networks that generalize well.

\section{Classification evaluation}

To test the classification algorithms, the 50 acquisitions/class database is first split in 40 acquisitions training dataset and 10 acquisitions generalisation dataset. To evaluate accurately the classifications rates we performed a 10fold cross-validation on the training dataset [14]. The basic processing steps can be summed up as follows:

1) separate the feature dataset into 10 folds per class

2) use 9 folds for training 
TABLE II

SPECTRUM CLASSIFICATION RATE OF 10 DIFFERENT PAPERS

\begin{tabular}{|c|c|c|c|}
\hline & Training & Validation & Test \\
\hline Original spectrum & $67.5 \pm 0.7 \%$ & $58 \pm 2 \%$ & $53 \pm 5 \%$ \\
\hline Centered spectrum & $71.8 \pm 0.7 \%$ & $60 \pm 2 \%$ & $61 \pm 5 \%$ \\
\hline
\end{tabular}

3) use the remaining fold for testing

4) repeat the two previous steps until all folds are used

5) store the average classification score

The different datasets are equally distributed between the 10 classes. But each MLP gives a one-against-rest output. To avoid an over training of the rest-class, the training dataset is partially replicated before entering each MLP. This way the binary output targets get equally distributed between the one-class and the rest-class. This replication is only applied during the training of MLP. It does not present any interest for classification evaluation, neither for the whole Euclidean classification (training nor evaluation). The original datasets as described above are used in these cases.

\section{E. Results}

1) Spectrum classification: We noted a quite important variance in decibel spectrum means between acquisition of the same class. Therefore, the classification algorithm was tested with original spectrum but also with centered spectrum (meanless decibel spectrum). Table II shows the classification results. In both cases, original or centered spectrum, we obtain good results with a classification rate of about $60 \%$ to be compared to the $10 \%$ classification rate of a random classification. As expected, the classification works slightly better on centered spectrum with more equilibrated results between validation and test.

2) 5 features classification: The spectra extracted from the signal are of large dimension (822 frequency components). With the aim of enlarging the database with much more samples of all sorts of material, it can be useful to reduce the number of features. The euclidean distance algorithm applied on the 5 features left gave worse results than spectrum classification. Taking into account non-linearities thanks to the neural network classification algorithm, we obtain results similar to spectrum classification but with much less features.

An essential parameter to be chosen in neural networks is the number of neurons in the hidden layer. A too large hidden layer will overfit the training dataset without increasing validation and test classification rates. The amount of data limits us to a maximum of 5 neurons. Figure 12 shows classification results of training and validation datasets as a function of the number of neurons. More than 2 neurons increases only the training classification rate giving then the limit over which the MLP is overfitting the training dataset. Table III gives the training, validation and test classification rates for the 10-class MLPs with 2 hidden neurons each.

The two hidden neuron classifier reaches a $71 \%$ validation rate but limits to $58 \%$ test rate. This difference between validation and test enhance the limit of classifier generalisation.

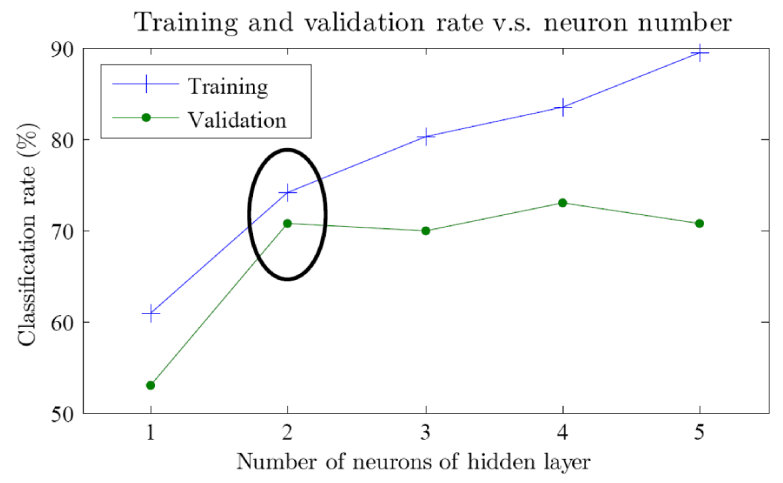

Fig. 12. Classification rates function of the number of neurons of the hidden layer. Note that the training classification rate increases continuously with number of hidden neurons while the validation rate stays constant over 2 hidden neurons. This is significant of overfitting over 2 hidden neurons.

TABLE III

10-CLASS WITH 2 HIDDEN NEURONS NETWORK CLASSIFICATION RATE

\begin{tabular}{|c|c|c|}
\hline Training & Validation & Test \\
\hline $74.1 \pm 0.7 \%$ & $71 \pm 2 \%$ & $58 \pm 5 \%$ \\
\hline
\end{tabular}

The training and validation datasets would need to be enlarged with the examples that are misclassified, and test dataset to be enlarge to better estimate the generalisation rate.

However, taking the $58 \%$ as a minimum classification rate of this last classifier, we can conclude to a similar and good performance of both spectrum-based and neural-network based algorithms. To compare the two algorithms, the differences between them are to be found in there performances for a particular application more than on these results. One major argument in favour of neural network is its ability to encircle classes by a non-linear limits. Therefore it should be more efficient as texture classes are getting more and more similar. One major drawback is a long training time due to the use of the whole database for MLPs weights training. Therefore, for a large and evolutive database of quite different textures, the euclidean distance algorithm would be more interesting.

We are now thinking of an algorithm merging the two precedent to increase the classification performances. At the moment, we are also working at increasing the sensitivity of our artificial finger prototype to enhance its texture perception and classification results.

\section{CONClusion}

During this study, we managed to package the three-axial force sensor as an artificial finger, with a quite biomimetic structure and size. Studying different shapes and hardness for the rubber-skin tested, we selected one that would be optimal for a dynamic texture recognition task. The characterisation of the artificial finger showed a great linearity in loading. For this study, we have dedicated the artificial finger to explore everyday life textures, like papers and fabrics. And the use of a plotter as an exploration system allowed us to automatize 
the exploration procedure and lead various kinds of texture recognition experiments.

In this paper we presented two preliminary texture exploration experiments that are attesting of the sensor sensitivity. The first one shows its ability to discriminate periodic or random coarse textures, for instance paper from fabric. The second one, with the reconstruction of the images of forces felt by the sensor, permitted to see the reproducibility of the measures, even with small events such as $10 \mu \mathrm{m}$ thick inked zones. Going further in texture grain size and recognition complexity, we finally evaluated the artificial finger with a multi-class discrimination experiment. For this tactile discrimination task we build up a sample set with 10 kinds of paper ((photo, drawing, blotting papers ...), a material commonly manipulated in every day life and which texture is usually tactily controlled for various reasons such as applications or marketing purposes. We explored these samples several times and applied different classification algorithms to the signal database acquired. One algorithm was based on euclidean distance to the mean spectrum, and another one on a neural network with 5 features as input. Both showed good results and testified of the sensor ability to discriminate fine textures. The next step will be to go beyond classification and characterize textures tactily, i.e. measuring softness, roughness, stickiness with the artificial finger. This opens up perspectives for various interesting applications.

\section{ACKNOWLEDGEMENT}

This work was partly supported by the EU-NEST programme, MONAT project (contract 21 number 29000). We would like to thank Patrice Rey (CEA-LETI) for providing the MEMS sensors necessary to make all our artifical finger prototypes and ArjoWiggins for providing Soft and Skin Touch paper samples. We would also like to thank J. Scheibert, A. Prevost and G. Debregeas (ENS Paris) for their very helpful advices.

\section{REFERENCES}

[1] M.E.H. Eltaib and J.R. Hewit, "Tactile sensing technology for minimal access surgery a review", Mechatronics, vol. 13, pp. 1163-1177, Dec. 2003.

[2] M. Tanaka, J.L. Lvque, H. Tagami , K. Kikuchi and S. Chonan, "The Haptic Finger - a new device for monitoring skin condition", Skin Res. Technol., vol. 9, pp. 131-136, May 2003.

[3] R. D. Howe and M. R.Curtosky, "Dynamic Tactile Sensing : Perception of Fine Surface Feature with stress rate Sensing", IEEE Trans. Robotics and Automation, Vol. 9, no. 2, pp. 140-151, Apr. 1993.

[4] M. Tanaka, H. Sugiura, J. L. Leveque, H. Tagami, K. Kikuchi and S. Chonan, "Active haptic sensation for monitoring skin conditions", $J$. Materials Process. Techno., Vol. 161, pp. 199-203, Apr. 2005.

[5] Y. Mukaibo, H. Shirado, M. Konyo and T. Maeno, "Development of a Texture Sensor Emulating the Tissue Structure and Perceptual Mechanism of Human Fingers", in Proc. 2005 IEEE Int. Conf. Robot. Autom., pp. 2565-2570.

[6] K. Hosoda, Y. Tada and M. Asada, "Anthropomorphic robotic soft fingertip with randomly distributed receptors", Robot. Auton. Syst., vol. 54, no. 2, pp. 104-109, Feb. 2006.

[7] L. Beccai et al., "Design and fabrication of a hybrid silicon three-axial force sensor for biomechanical applications", Sens. Actuators A, vol. 120, no. 2, pp. 370382, May 2005.
[8] B. J. Kane, M. R. Cutkosky and G. T. A. Kovacs, "A Traction Stress Sensor Array for Use in High-Resolution Robotic Tactile Imaging", $J$. Microelectromech. Syst., Vol. 9, no .4, Dec. 2000.

[9] K. Kim et al, "A silicon-based flexible tactile sensor for ubiquitous robot companion applications", 2006 J. Phys.: Conf. Ser. 34, pp. 399-403.

[10] G. Vasarhelyi et al., "Characterisation of an integrable single-crystalline 3-D Tactile Sensor", IEEE Sens. J., Vol. 6.4, pp. 928-934, Aug. 2006.

[11] L. Beccai et al., "Development and Experimental Analysis of a Soft Compliant Tactile Microsensor for Anthropomorphic Artificial Hand", IEEE/ASME Trans. Mechatronics, vol. 13, pp. 158-168, Apr. 2008.

[12] C.T. Yao, M.C. Peckerar, J.H. Wasilik, C. Amazeen and S. Bishop, "A novel three-dimensional microstructure fabrication technique for a triaxial tactile sensor array", in Proc. IEEE Micro Robot. Teleop. Workshop, 1987.

[13] M. Hollins and S. J. Bensmaia, "The coding of roughness", Can. J. Exp. Psychol., vol. 61, pp. 184-95, Sep. 2007.

[14] R. O. Duda, P. E. Hart and D. G. Stork, "Cross-Validation", in Pattern Recognition, 2nd ed., Wiley Interscience, 2001, pp. 483-484. 